\title{
Validação de dados termopluviométricos obtidos via sensoriamento remoto para o Estado de São Paulo
}

\author{
Ludmila B. Camparotto', Gabriel C. Blain ${ }^{1}$, Angélica Giarolla², \\ Marcos Adami ${ }^{3}$ \& Marcelo B. P. de Camargo ${ }^{1}$
}

\begin{abstract}
RESUMO
Dados termopluviométricos são utilizados em diversos estudos. Contudo, a escassez de dados resultante da baixa densidade de estações meteorológicas é um problema frequentemente encontrado. Com isto, a utilização de dados estimados por satélites tem-se mostrado importante para regiões nas quais eles são escassos. Neste trabalho foram realizadas comparações entre dados mensais de precipitação pluvial estimados pelo satélite TRMM e de temperatura, estimados pelo satélite Aqua/MODIS aos dados observados por estações de superfície, para diferentes localidades do Estado de São Paulo, no período de 2003 a 2010. Os resultados demonstraram que para os dados de precipitação pluvial os menores erros foram observados nos meses de inverno e os maiores nos meses de verão. Em relação aos dados de temperatura observou-se que, com a inclusão de um fator de correção, os dados do satélite Aqua apresentaram boa exatidão em todos os meses. De maneira geral, os resultados obtidos indicam que os satélites TRMM e Aqua são alternativas eficientes na aquisição de informações em locais onde há falhas ou escassez dos mesmos podendo ser também utilizados na análise da consistência de dados coletados por meio de redes meteorológicas.
\end{abstract}

Palavras-chave: TRMM, Aqua/MODIS, temperatura do ar, precipitação pluvial

\section{Validation of temperature and rainfall data obtained by remote sensing for the State of Sao Paulo, Brazil}

\begin{abstract}
Temperature and rainfall data are used in several scientific studies. However, the paucity of data resulting from the low density of weather stations is a problem frequently encountered in these studies. Thus, the use of estimated data from satellites has become an important tool in regions where they are scarce. In this study comparisons were made between monthly rainfall data estimated by TRMM satellite and temperature data estimated by the satellite Aqua/MODIS data observed by surface stations, for different locations in the State of Sao Paulo during the period 2003 to 2010. The results showed that for the rainfall data, smaller errors were observed during the winter and higher during the summer months. In relation to the temperature data it was observed that with the inclusion of the correction factor, the Aqua satellite data showed good accuracy in all months. Overall, the results obtained by validation of the data of rain and temperature, indicating that the satellite TRMM and Aqua are efficient alternatives for obtaining information of locations where there are errors or shortage of the same, may also be used to analyse the consistency of data collected by meteorological networks.
\end{abstract}

Key words: TRMM, Aqua/MODIS, air temperature, rain

' IAC/APTA/SAA, Centro de Ecofisiologia e Biofísica. CP 28, CEP 13001-970, Campinas, SP. Fone: (19) 3202-1689. E-mail: ludmila_bardin@yahoo.com.br; gabriel@iac.sp.gov.br; mcamargo@iac.sp.gov.br

2 CCST/INPE. CEP 12227-010, São José dos Campos, SP. Fone: (12) 3208-7123. E-mail: angelica.giarolla@inpe.br

${ }^{3}$ INPE, Centro Regional da Amazônia. CEP 66077-830, Belém, PA. Fone: (91) 3032-5156. E-mail: marcos.adami@inpe.br 


\section{INTRODUÇÃO}

As estações meteorológicas fornecem registros pontuais, frequentemente sujeitos a falhas que limitam o uso dessa informação. A baixa densidade de pluviômetros, a longa distância e a distribuição irregular das estações não permitem, devido ao seu caráter pontual, capturar a variabilidade espacial da precipitação pluvial, gerando incertezas nos resultados de diversos estudos aplicados no País.

Camargo et al. (2005) quantificaram a variabilidade espacial e temporal de dados diários termopluviométricos para o Estado de São Paulo e verificaram que, à medida em que aumentam as distâncias, os valores de coeficiente de determinação, obtidos a partir da comparação entre registros de distintos postos, diminuem e os erros também aumentam. Diversos trabalhos nas áreas de planejamento urbano, previsão de tempo, gestão de recursos hídricos e impactos ao meio ambiente e clima, comparando-se dados de precipitação entre satélites e estações meteorológicas, vêm sendo desenvolvidos (Anagnostou et al., 2001; Sherptterd et al., 2002; Collischonn et al., 2008).

Embora a estimativa da precipitação pluvial a partir de sensores orbitais utilizando instrumento de radar se tenha iniciado nos anos 80, a credibilidade dessas medidas ainda é um tema recorrentemente avaliado. O satélite Tropical Rainfall Measuring Mission (TRMM)foi lançado em novembro de 1997 visando monitorar e estudar a precipitação nos trópicos, além de verificar como a mesma influencia o clima global (Kummerow et al., 2000). Segundo Barrera (2005) este é o satélite mais bem equipado em termos de instrumentos para estimativa de precipitação, fornecendo dados mais precisos do que técnicas indiretas, baseadas em imagens de outros satélites. Entretanto, ainda são necessários estudos que validem essas informações, visando quantificar sua precisão e estabelecer uma aplicação direta desses produtos (Kummerow et al., 2000; Kurtzman et al., 2009; Karaseva et al., 2011).

Neste contexto, diversos trabalhos foram desenvolvidos visando validar ou comparar as estimativas da precipitação pluvial por satélite em diferentes regiões; tais como: Feidas et al. (2010) para a Grécia e Javanmard et al. (2010) para o Irã. Karaseva et al. (2011) realizaram a validação dos dados mensais obtidos via sensoriamento remoto para o Quirguistão, enfocando a verificação da precisão em altas latitudes e em regiões com orografia complexa. Ressalta-se que em países desenvolvidos (EUA e Europa) poucos estudos são desenvolvidos nesta área em virtude da densa rede de estações meteorológicas existente. No Brasil, Collischonn et al. (2007) realizaram a validação para a bacia do Paraguai Superior e mostraram que, no caso de escassez de dados, os valores estimados pelo satélite podem ser utilizados. Para o Estado de São Paulo resultados preliminares indicaram que as estimativas da precipitação pluvial fornecidas pelo satélite TRMM conseguiram reproduzir com consistência os totais descendais de precipitação pluvial na região da Alta Mogiana (Bardin et al., 2010).

Em 2002 foi lançado o satélite Aqua, pela NASA, abordo do qual está seu principal instrumento, o sensor Moderate Resolution Imaging Spectroradiometer (MODIS), projetado para fornecer uma série de observações globais da superfície terrestre, oceano e atmosfera nas regiões do visível e do infravermelho do espectro eletromagnético, cobrindo a Terra a cada dois dias (Latorre et al., 2003). O produto MYD11 é uma das variáveis-chave para diversos estudos climáticos, hidrológicos, ecológicos e biológicos (Running et al., 1994).

Alguns trabalhos envolvendo imagens de satélites estão sendo desenvolvidos na área da agrometeorologia visando, sobremaneira, comparar dados de temperatura de superfície, estimados por satélites e dados obtidos em estações meteorológicas (Souza \& Silva, 2005; Picoli et al., 2009; Tomlinson et al., 2012; Lin et al., 2012). Ataíde et al. (2009) avaliaram dados de temperatura de superfície estimados pelo sensor MODIS e valores de estações meteorológicas para o Estado de Goiás e observaram que a diferença entre os dados foi, em média, de $7,6^{\circ} \mathrm{C}$, sendo conveniente a aplicação de um fator de correção linear. Klering (2007) avaliou o uso de imagens do MODIS na modelagem agrometeorológica-espectral de rendimento de arroz irrigado no Rio Grande do Sul, concluindo tendência de melhoria nas estimativas, a partir da incorporação de dados provenientes das imagens do MODIS.

Desta forma, torna-se evidente que as estimativas de dados termopluviométricos via sensoriamento remoto se constituem numa ferramenta extremamente útil que visa melhorar a rede de obtenção de informações meteorológicas, além se ser importantes no auxílio de preenchimento de dados faltosos. Neste contexto, o objetivo do trabalho foi validar o uso dessas estimativas de precipitação pluvial e temperatura do ar no Estado de São Paulo.

\section{Material e Métodos}

Obtiveram-se dados mensais de precipitação pluvial por meio do satélite TRMM e de temperatura, pelo satélite Aqua/ MODIS. Esses dados foram comparados aos dados pontuais de superfícies oriundos de 30 estações meteorológicas pertencentes ao Instituto Agronômico (IAC/CIIAGRO). O período para comparação foi de oito anos (2003 a 2010). Os dados do TRMM e MODIS foram agrupados e analisados na escala mensal e os testes estatísticos foram realizados visando verificar a exatidão e a precisão dessas estimativas. Na Figura 1 são apresentadas as localizações das estações utilizadas.

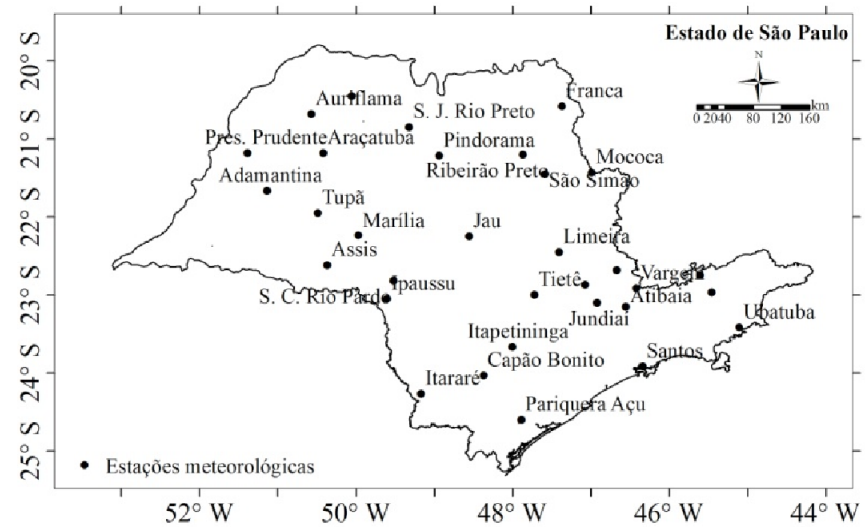

Figura 1. Localização das estações meteorológicas utilizadas nas comparações com dados via sensoriamento remoto 
O TRMM gera diferentes produtos ou estimativas de acordo com a combinação de instrumentos utilizados no algoritmo de cálculo. Os dados de precipitação pluvial foram obtidos do produto 3B42 (Fonte: http://trmm.gsfc.nasa.gov) que utiliza estimativas de precipitação por micro-ondas do imageador de micro-ondas (TMI) corrigidas por informações da estrutura vertical das nuvens obtidas do radar de precipitação (PR). Os dados foram fornecidos em $\mathrm{mm} \mathrm{h}^{-1} \mathrm{em}$ arquivos raster com extensão hdf (hierarchical data format) e resolução espacial de $0,25^{\circ}$ (cerca de $25 \mathrm{~km}$ ).

Para cada estação de superfície existem quatro pontos no seu entorno, referentes aos pontos do TRMM. Para comparação entre os dados obtidos via sensoriamento remoto e de superfície foram extraídos, das imagens, os valores dos pontos do TRMM mais próximos aos pluviômetros, conforme esquematizado na Figura 2.

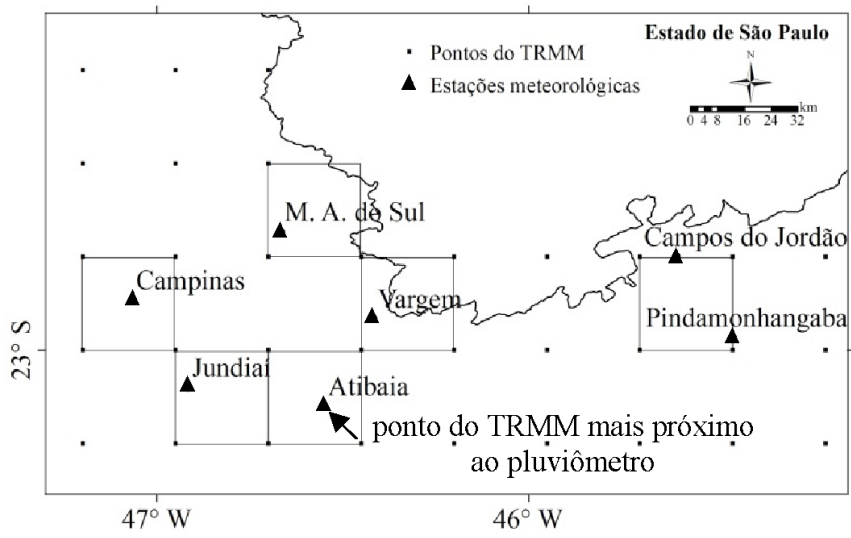

Figura 2. Exemplificação da localização de algumas estações meteorológicas e dos pontos (TRMM) utilizados na comparação de dados pluviométricos estimados e observados

Os dados de temperatura de superfície estimados foram obtidos a partir do produto MYD11, sensor MODIS do satélite Aqua, no formato hdf (Fonte:http://modis-land.gsfc.nasa.gov/ temp.htm - MODIS/AquaLand SurfaceTemperature/Emissivity Monthly L3 Global 0.05Deg CMG) com resolução espacial de aproximadamente $5,6 \mathrm{~km}$. Nas imagens obtidas por meio do sensor MODIS foi utilizado o programa de geoprocessamento ENVI (Environment for Visualizing Images) para conversão dos valores digitais da imagem para Kelvin, multiplicando-se cada pixel por um fator de ajuste de escala igual a 0.02 (Wan, 1999); posteriormente, os valores foram transformados para graus Celsius.

A temperatura média do satélite (Tms) foi gerada com base nas duas passagens diárias sobre o meridiano local (aproximadamente 3 e 13 h e $30 \mathrm{~min}$ ). Para comparação entre os dados obtidos via sensoriamento remoto e de superfície foram extraídos, das imagens, os valores dos pixels nos quais se encontram inseridos os abrigos meteorológicos, conforme esquematizado na Figura 3.

Ressalta-se que a temperatura média das estações meteorológicas é baseada em dados de temperatura máxima e mínima, as quais podem ocorrer em horários distintos da passagem do satélite. Com isto, foi necessária uma correção

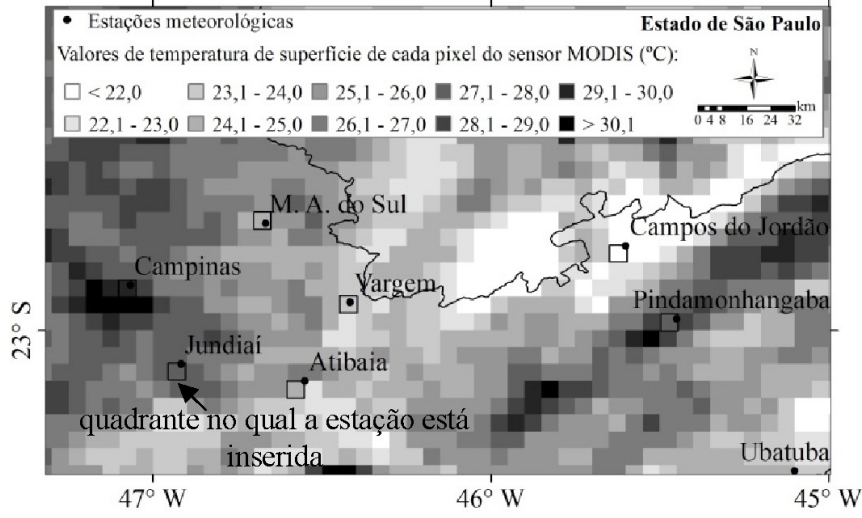

Figura 3. Exemplificação da localização das estações meteorológicas e dos pixels utilizados na comparação de dados de temperatura estimados e observados

de Tms realizada com base nos coeficientes da regressão linear simples entre os dados de superfície e do satélite. A estimativa desses parâmetros foi realizada para os anos de 2003 a 2008. A validação de Tms baseou-se nos anos de 2009 e 2010.

As análises estatísticas foram realizadas visando verificar a exatidão e a precisão dos dados de sensoriamento remoto. Ressalta-se que a exatidão se refere ao grau de conformidade de um valor estimado em relação ao valor verdadeiro (observado). A precisão é o grau de variação de resultados de uma medição e tem como base, o desvio-padrão de uma série de repetições da mesma análise.

De acordo com Wilks (2006) o erro médio (EM), o erro médio absoluto (EMA) e o erro quadrado médio (EMQ) são métodos bastante apropriados para verificação da acurácia ou exatidão escalar de dados estimados (e) em relação aos observados (o) em uma amostra com $\mathrm{N}$ pares de i de dados, sendo descritos por:

$$
\mathrm{EM}=\mathrm{N}^{-1} \sum_{\mathrm{i}=1}^{\mathrm{N}} \mathrm{o}_{\mathrm{i}}-\mathrm{e}_{\mathrm{i}}
$$

Analisando a Eq. 1 verifica-se que diferenças positivas (o $>$ e) tendem a anular diferenças negativas $(\mathrm{o}<\mathrm{e})$. A fim de evitar esse possível erro, utilizam-se os valores absolutos das diferenças (Eq. 2):

$$
\mathrm{EMA}=\mathrm{N}^{-1} \sum_{\mathrm{i}=1}^{\mathrm{N}}\left|\mathrm{o}_{\mathrm{i}}-\mathrm{e}_{\mathrm{i}}\right|
$$

A Eq. 3 pode, segundo Wilks (2006), ser interpretada como a magnitude do erro inerente à estimativa de uma amostra de dados.

$$
\mathrm{EMQ}=\mathrm{N}^{-1} \sum_{\mathrm{i}=1}^{\mathrm{N}}\left(\mathrm{o}_{\mathrm{i}}-\mathrm{e}_{\mathrm{i}}\right)^{2}
$$

As Eqs. 2 e 3 são similares. Contudo, devido ao fato de utilizar a potência de dois, o EMQ é mais sensível às maiores diferenças entre os dados observados e os estimados.

A análise de regressão linear simples foi utilizada fornecendo valores do intercepto (A) e do coeficiente angular (B). O 
coeficiente de correlação (r) também foi utilizado (significativo a 90\%). Para verificação da qualidade final do modelo estimador foi utilizado o método proposto por Willmott (1981), índice de concordância também chamado índice "d" de Willmott. Este método pode ser calculado pelas seguintes equações:

$$
\mathrm{d}_{2}=1-\left[\frac{\sum\left(\mathrm{e}_{\mathrm{i}}-\mathrm{o}_{\mathrm{i}}\right)^{2}}{\sum\left(\left|\mathrm{e}_{\mathrm{i}}-\mathrm{o}\right|+\left|\mathrm{o}_{\mathrm{i}}-\mathrm{o}\right|\right)^{2}}\right]
$$

em que:

$$
\begin{array}{ll}
\mathrm{d}_{2} & \text { - índice de concordância } \\
\mathrm{o} & \text { - dados observados } \\
\mathrm{e} & \text { - são os dados estimados }
\end{array}
$$

Embora a estatística $\mathrm{d}_{2}$, conforme descrita por Willmott (1981) seja largamente utilizada (Moraes et al., 1998; Gonçalves et al., 2008; Bardin et al., 2010). Willmott et al. (1985) e Legates \& Mccabe (1999) descreveram que a utilização da função quadrática na Eq. 4 pode resultar em valores elevados deste índice, mesmo quando não há um bom desempenho do modelo estimador. Desta forma, Willmott et al. (1985) propõem uma adaptação da Eq. 4, denominada índice de Willmott modificado, dado por:

$$
\mathrm{d}_{1}=1-\left[\frac{\sum\left(\mathrm{e}_{\mathrm{i}}-\mathrm{o}_{\mathrm{i}}\right)}{\sum\left(\left|\mathrm{e}_{\mathrm{i}}-\mathrm{o}\right|+\left|\mathrm{o}_{\mathrm{i}}-\mathrm{o}\right|\right)}\right]
$$

em que:

$\mathrm{d}_{1} \quad$ - índice de concordância modificado

o - são os dados observados; e são os dados estimados

Segundo Legates \& Mccabe (1999) a vantagem da Eq. 5 , em relação à Eq. 4, se deve ao fato de que os erros $\left(\mathrm{e}_{\mathrm{i}}-\mathrm{o}_{\mathrm{i}}\right)$ não são influenciados pela potência de dois, o que, segundo esses autores, resulta em um índice mais rigoroso visto que os valores de $d_{1}$ tendem a ser inferiores aos de $d_{2}$. Os valores de $d_{1}$ e $\mathrm{d}_{2}$ variam entre 0 e 1 , sendo que valores próximos à unidade indicam bom ajuste.

De acordo com Willmott et al. (1985) após estimar as medidas estatísticas de precisão e exatidão, aqui representadas pelo vetor $\theta=\left[E M A, E M Q, r, d_{1}\right.$ e $d_{2}$ ], é interessante determinar o intervalo sob o qual se espera que os valores de $\theta$ estejam contidos. Segundo esses autores, a magnitude deste intervalo de confiança pode ser vista como medida da confiabilidade de $\theta$. Neste aspecto, ressalta-se que os limites superior (b) e inferior (a) de um intervalo de confiança podem ser descritos por:

$$
\int_{a}^{b} f(\theta) d \theta=P\{a<\theta<b\}=1-\alpha
$$

em que:

$\theta$ assume os valores de EMA, EMQ, $r, d_{1}$ ou $d_{2}$

$\mathrm{P}\{\mathrm{a}<\theta<\mathrm{b}\}$ - probabilidade de que $\theta$ esteja contido no intervalo [a,b]

$\alpha \quad$ - um valor entre 0 e 1 (usualmente igualado a 0,05 )

$\mathrm{f}(\theta)$ - função densidade de probabilidade que descreve a população da qual os valores de $\theta$ são oriundos
Quando $f(\theta)$ é conhecido, a solução da Eq.6 pode ser facilmente calculada; contudo, quando $\mathrm{f}(\theta)$ é desconhecida, como no presente estudo, Efron (1981a,b) e Efron \& Gong (1983) sugerem que o método não paramétrico de permutação denominado bootstrap seja utilizado na determinação do referido intervalo de confiança.

O bootstrap constrói distribuições empíricas da medida em análise permitindo a determinação do intervalo de confiança associada à mesma (Willmott et al., 1985). No presente trabalho foram construídas 10.000 séries sintéticas. Descrições detalhadas sobre o bootstrap podem ser encontradas em Willmott et al. (1985) e Wilks (2006).

\section{Resultados e Discussão}

Na Tabela 1 são apresentados os resultados das análises realizadas entre os dados de precipitação pluvial, obtidos em estações de superfície e estimados pelo satélite TRMM. De forma geral, observa-se que os maiores valores de EMA ocorreram nos meses de dezembro, janeiro e fevereiro. $\mathrm{O}$ maior valor foi igual a $81,6 \mathrm{~mm}$ (janeiro). Esta característica pode ser explicada devido às chuvas convectivas, geralmente de forte intensidade e que ocorrem de forma pontual.

Nos meses de inverno os valores de EMA foram mais baixos, sendo o menor valor observado no mês de junho (13,7 mm). Nesta estação as precipitações pluviais são mais generalizadas, de baixa intensidade, frequentemente causadas por entrada de massas de ar frio, que cobrem uma grande região. Ressalta-se que os valores de $d_{1}$ e $d_{2}$ mais elevados se concentraram nos meses de julho, agosto e setembro. Assim como observado por Collischonn et al. (2007), Karaseva et al. (2011) e Li et al. (2011) os resultados mostraram que as estimativas de precipitação fornecidas pelo TRMM são consistentes porém alguns erros ainda existem e são discrepantes podendo ocorrer devido pelo fato do TRMM abranger uma área de $25 \times 25 \mathrm{~km}$ enquanto a estação meteorológica mede a precipitação, de forma pontual.

Os valores apresentados na Tabela 2 permitem inferir que, inicialmente, as temperaturas obtidas por meio do satélite Aqua (anteriores ao ajuste proposto) se aproximam mais dos dados de superfície nos meses de inverno. Esta inferência se baseia nos valores do coeficiente angular próximos à unidade, indicando inclinação similar à reta $1: 1$, tal como nos menores valores do intercepto obtidos nesses quatro meses.

A validação do modelo com base nos anos de 2009 e 2010 é apresentada na Tabela 3 . Os valores de $d_{1}$ e $d_{2}$ corroboram com a inferência realizada com base na Tabela 2 , de vez que os valores mais elevados dessas medidas exatidão tendem a se concentrar nos meses de junho, julho e agosto. A análise da Tabela 3 concorda com a indicação de Legates \& Mccabe (1999) no sentido de que os valores de $\mathrm{d}_{1}$ tendem a ser inferiores aos de $\mathrm{d}_{2}$.

De forma geral e após o ajuste, observa-se que os dados do satélite apresentaram boa exatidão em todos os meses. Ressalta-se que o maior valor de EMA foi igual a $1,30^{\circ} \mathrm{C}$ (novembro; Tabela 3). Ataíde et al. (2009) também observaram a necessidade de aplicação de um fator de correção nos dados obtidos pelo satélite MODIS. Após a aplicação do fator de correção os resultados obtidos por esses autores elevaram a aproximação de 69 para $90 \%$ entre os valores medidos e os estimados 
Tabela 1. Validação dos dados de precipitação pluvial do satélite TRMM: erro médio absoluto (EMA), erro médio quadrático (EMQ), os índices de concordância de Willmott (d2) e Willmott modificado (d1) e o coeficiente linear de correlação $(r)^{*}$

\begin{tabular}{cccccc}
\hline Mês & EMA (mm) & EMQ (mm $\mathbf{~ m}^{2}$ & $\mathbf{d 1}$ & $\mathbf{d}$ & $\mathbf{r}$ \\
Jan & $81,61[73,71: 88,94]$ & $101,87[91,57: 111,44]$ & $0,49[0,45: 0,54]$ & $0,71[0,65: 0,76]$ & $0,52[0,40: 0,63]$ \\
Fev & $60,24[54,59: 66,25]$ & $76,23[69,21: 83,28]$ & $0,56[0,51: 0,60]$ & $0,78[0,73: 0,82]$ & $0,68[0,59: 0,77]$ \\
Mar & $47,69[42,93: 52,72]$ & $61,21[54,84: 67,89]$ & $0,54[0,49: 0,58]$ & $0,77[0,70: 0,81]$ & $0,62[0,51: 0,74]$ \\
Abr & $36,70[33,12: 40,42]$ & $47,28[43,10: 51,72]$ & $0,54[0,49: 0,58]$ & $0,75[0,70: 0,79]$ & $0,61[0,51: 0,71]$ \\
Mai & $21,48[18,64: 24,56]$ & $31,61[26,54: 36,62]$ & $0,71[0,67: 0,74]$ & $0,89[0,85: 0,92]$ & $0,80[0,74: 0,87]$ \\
Jun & $13,68[11,99: 15,48]$ & $19,58[16,80: 22,24]$ & $0,67[0,63: 0,71]$ & $0,83[0,79: 0,87]$ & $0,69[0,60: 0,78]$ \\
Jul & $18,32[15,57: 21,27]$ & $28,97[23,80: 34,18]$ & $0,81[0,77: 0,83]$ & $0,95[0,93: 0,96]$ & $0,90[0,87: 0,94]$ \\
Ago & $15,95[13,30: 19,01]$ & $28,30[22,09: 34,97]$ & $0,74[0,69: 0,79]$ & $0,89[0,84: 0,93]$ & $0,82[0,74: 0,90]$ \\
Set & $26,76[22,45: 32,16]$ & $45,36[33,96: 59,29]$ & $0,74[0,70: 0,78]$ & $0,89[0,85: 0,93]$ & $0,80[0,73: 0,88]$ \\
Out & $38,66[34,65: 42,82]$ & $49,91[44,90: 55,12]$ & $0,55[0,49: 0,60]$ & $0,76[0,70: 0,82]$ & $0,61[0,49: 0,72]$ \\
Nov & $46,34[41,73: 50,97]$ & $58,18[52,82: 63,55]$ & $0,57[0,52: 0,61]$ & $0,80[0,74: 0,84]$ & $0,66[0,56: 0,75]$ \\
Dez & $64,23[57,85: 70,51]$ & $81,97[74,88: 89,11]$ & $0,57[0,52: 0,62]$ & $0,78[0,73: 0,83]$ & $0,64[0,54: 0,74]$ \\
\hline
\end{tabular}

* Medidas estatísticas foram obtidas a partir da comparação entre dados de precipitação pluvial estimados do satélite TRMM e observados em estações meteorológicas, para os anos de 2003 a 2010

Tabela 2. Coeficientes da equação de regressão linear simples, intercepto (a) e coeficiente angular (b), utilizados no ajuste dos dados de temperatura do satélite Aqua, sensor MODIS

\begin{tabular}{|c|c|c|c|c|c|c|c|c|c|c|c|c|}
\hline \multirow{2}{*}{ Parâmetro } & \multicolumn{12}{|c|}{ Meses } \\
\hline & Jan & Fev & Mar & Abr & Mai & Jun & Jul & Ago & Set & Out & Nov & Dez \\
\hline$b^{\star}$ & 0,59 & 0,77 & 0,79 & 0,83 & 0,82 & 0,93 & 0,92 & 0,84 & 0,74 & 0,62 & 0,63 & 0,68 \\
\hline
\end{tabular}

* Desenvolvido com base no período 2003 a 2008, para o Estado de São Paulo

Tabela 3. Validação dos dados de temperatura do satélite Aqua, sensor MODIS: erro médio absoluto (EMA), erro médio quadrático (EMQ) e os índices de concordância de Willmott (d2) e Willmott modificado (d1) e o coeficiente linear de correlação $(r)^{*}$

\begin{tabular}{cccccc}
\hline Mês & EMA $\left({ }^{\circ} \mathbf{C}\right)$ & EMQ $\left(^{0} \mathbf{C}^{2}\right)$ & $\mathbf{d 1}$ & $\mathbf{d}$ & $\mathbf{r}$ \\
Jan & $1,11[0,88: 1,35]$ & $1,43[1,15: 1,69]$ & $0,57[0,46: 0,66]$ & $0,80[0,69: 0,87]$ & $0,59[0,34: 0,84]$ \\
Fev & $0,92[0,71: 1,13]$ & $1,23[0,98: 1,48]$ & $0,67[0,57: 0,75]$ & $0,88[0,79: 0,92]$ & $0,73[0,58: 0,89]$ \\
Mar & $0,99[0,83: 1,18]$ & $1,20[1,01: 1,43]$ & $0,62[0,50: 0,70]$ & $0,88[0,76: 0,92]$ & $0,73[0,55: 0,91]$ \\
Abr & $1,02[0,79: 1,24]$ & $1,35[1,06: 1,62]$ & $0,67[0,58: 0,74]$ & $0,88[0,78: 0,92]$ & $0,72[0,55: 0,90]$ \\
Mai & $0,99[0,79: 1,19]$ & $1,25[1,03: 1,46]$ & $0,67[0,58: 0,74]$ & $0,90[0,81: 0,93]$ & $0,86[0,77: 0,95]$ \\
Jun & $1,02[0,83: 1,20]$ & $1,27[1,05: 1,45]$ & $0,72[0,64: 0,78]$ & $0,92[0,88: 0,94]$ & $0,88[0,82: 0,94]$ \\
Jul & $0,98[0,77: 1,18]$ & $1,27[1,01: 1,49]$ & $0,74[0,66: 0,80]$ & $0,93[0,88: 0,96]$ & $0,87[0,80: 0,94]$ \\
Ago & $1,00[0,83: 1,18]$ & $1,23[1,03: 1,42]$ & $0,73[0,67: 0,78]$ & $0,92[0,89: 0,94]$ & $0,85[0,78: 0,93]$ \\
Set & $1,05[0,87: 1,25]$ & $1,30[1,08: 1,51]$ & $0,72[0,63: 0,78]$ & $0,91[0,87: 0,94]$ & $0,83[0,74: 0,91]$ \\
Out & $1,11[0,92: 1,31]$ & $1,37[1,15: 1,60]$ & $0,67[0,61: 0,73]$ & $0,88[0,84: 0,91]$ & $0,81[0,71: 0,91]$ \\
Nov & $1,30[1,08: 1,54]$ & $1,60[1,36: 1,86]$ & $0,57[0,46: 0,65]$ & $0,83[0,72: 0,89]$ & $0,72[0,54: 0,90]$ \\
Dez & $1,08[0,86: 1,31]$ & $1,40[1,13: 1,66]$ & $0,59[0,48: 0,69]$ & $0,84[0,71: 0,90]$ & $0,65[0,45: 0,85]$ \\
\hline
\end{tabular}

* Medidas estatísticas foram obtidas a partir da comparação entre dados de superfície e do satélite Aqua

Na Figura 4 são exemplificadas, para as localidades de Franca, Campinas, Marília e Votuporanga, as comparações entre os valores observados de temperatura e os ajustados por meio dos parâmetros apresentados na Tabela 2. As análises visuais da Figura 4 corroboram com os resultados apresentados na Tabela 3, no sentido de que o satélite Aqua é capaz de fornecer boas estimativas da temperatura observada em superfície. $\mathrm{O}$ município de Franca foi o que apresentou o melhor resultado. Após a correção dos valores estimados observa-se que estes estão muito próximos aos observados nas estações de superfície.

O município de Campinas foi o que apresentou pior desempenho, pois os valores estimados, mesmo após a aplicação das equações de correção, continuam superestimados em relação aos dados observados, ao contrário dos demais, este último posto meteorológico está localizado no centro experimental do Instituto Agronômico, situado na região metropolitana do município de Campinas, evento que faz com que o pixel que engloba esse ponto de observação superficial compreenda diversos tipos de ocupação do solo, tais como rodovias, edificações urbanas e, naturalmente, o próprio parque experimental do IAC, no qual podem ser encontradas superfícies com diversas coberturas vegetais e lagos. Futuros estudos poderão verificar a hipótese de que essa característica resulta na maior diferença entre a temperatura média do pixel e a observada no posto meteorológico (Figura 4). É também importante observar que, mesmo quando não há um ajuste perfeito entre as curvas apresentadas na Figura 4, elas sempre mantêm a mesma tendência. Em outras palavras, a elevação (queda) da temperatura em superfície é sempre acompanhada pelo aumento (diminuição) das temperaturas obtidas pelo satélite.

Assim como observado por diferentes autores (Collischonn et al., 2007; Karaseva et al., 2011) os resultados obtidos por meio da validação dos dados de precipitação pluvial e de temperatura, indicam que os satélites TRMM e Aqua são alternativas eficientes para aquisição de informações em locais do Estado 


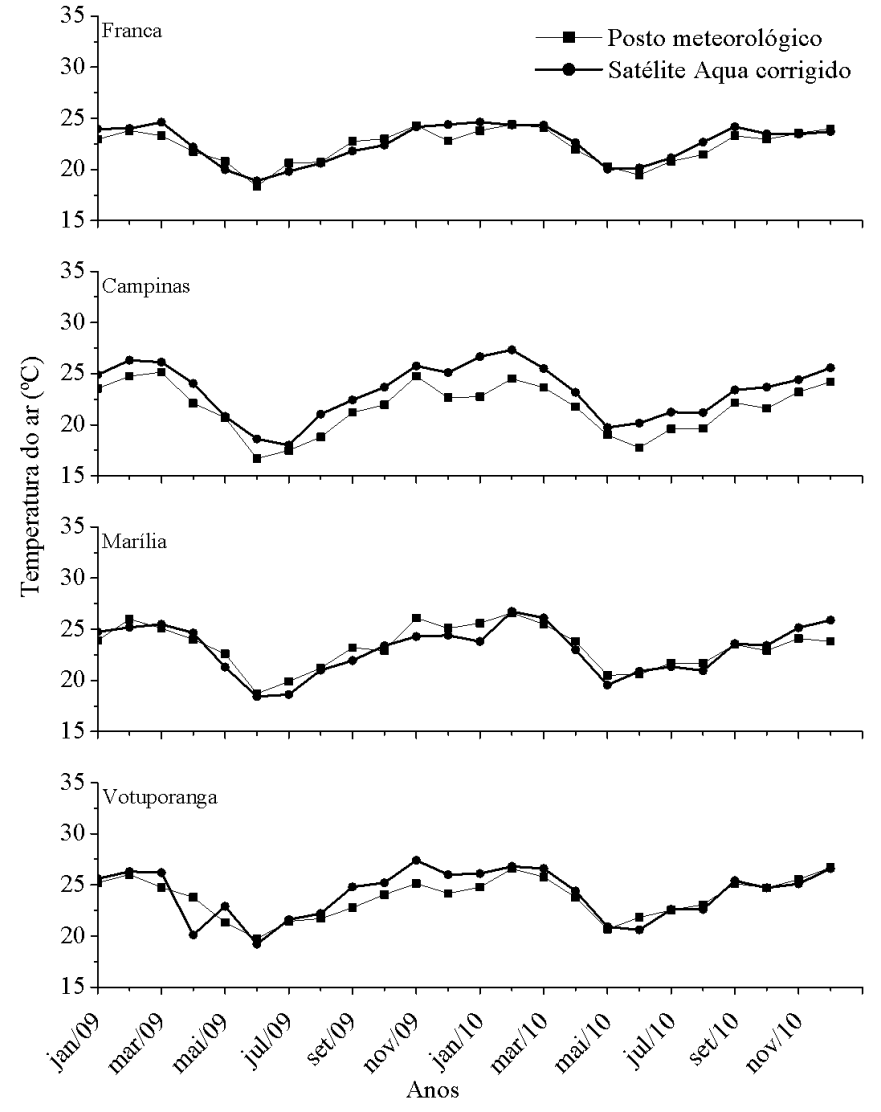

Figura 4. Comparação entre os dados observados pelas estações meteorológicas, dados de temperatura média de superfície estimados do satélite Aqua e dados do satélite Aqua corrigidos, para os anos de 2009 e 2010

de São Paulo onde ocorrem falhas ou escassez dos mesmos, podendo ser também utilizados na análise da consistência de valores obtidos por meio de redes meteorológicas.

\section{Conclusões}

1. As estimativas de precipitação pluvial obtidas do satélite TRMM, apresentaram maiores erros nos meses de verão em detrimento da sua grande variabilidade; nas demais estações do ano, os erros foram menores.

2. Para os dados de temperatura média estimada (satélite Aqua/MODIS) e em função dos diferentes horários de passagem do satélite, não apresentaram boa exatidão e precisão. Com isto, tornou-se conveniente a aplicação de um fator de correção que resultasse em dados com maior consistência.

3. Os resultados obtidos por meio da validação dos dados de precipitação pluvial e de temperatura indicaram que as estimativas fornecidas pelos satélites TRMM e Aqua são alternativas eficientes na aquisição dessas informações no Estado de São Paulo. Tais estimativas podem ser também utilizadas nas análises de consistência de valores obtidos em estações de superfície.

\section{Agradecimentos}

À Fundação de Amparo à Pesquisa do Estado de São Paulo (FAPESP) pela concessão da bolsa de Pós-graduação (Doutorado) para Ludmila Bardin Camparotto.

\section{Literatura Citada}

Anagnostou E. N.; Morales, C. A.; Dinku, T. The use of TRMM precipitation radar observations in determining ground radar calibration biases. Journal of Atmospheric and OceanicTechnology, v.18, p.616-628, 2001.

Ataíde, K. R. P.; Ledo, I. D.; Oliveira, M. G. R.; Bezerra, W. A. Avaliação da estimativa da temperatura de superfície obtida pelo sensor MODIS para o estado de Goiás. Natal, Brasil. In: Simpósio Brasileiro de Sensoriamento Remoto, 14, 2009. Natal. Anais... Natal: INPE, 2009. CD-Rom

Bardin, L.; Camargo, M. B. P.; Blain, G. C.; Silva, D. F. Comparação entre dados de precipitação pluvial observados em estações meteorológicas e estimados pelo satélite TRMM para a região cafeeira da Mogiana, São Paulo, Brasil. In: Reunión Argentina, 13, e Latinoamericana de Agrometeorología, 2010, Bahía Blanca. Anais... Bahía Blanca: RALDA, 2010. CD-Rom

Barrera, D. F. Precipitation estimation with the hydro-estimator technique: its validation against raingage observations. In: Congresso da IAHS, 7, 2005, Foz do Iguaçu. Anais...Foz do Iguaçu: IAHS, 2005. CD-Rom

Camargo, M. B. P.; Brunini, O.; Pedro Júnior, M. J.; Bardin, L. Variabilidade espacial e temporal de dados termopluviométricos diários da rede de estações agrometeorológicas do Instituto Agronômico (IAC). Bragantia, v.64, p.473-483, 2005.

Collichonn, B.; Allasia, D.; Collishonn, W.; Tucci, C. E. M. Desempenho do satélite TRMM na estimativa de precipitação sobre a bacia do Paraguai Superior. Revista Brasileira de Cartografia, v.59, p.93-99, 2007.

Collischonn, B.; Collischonn, W.; Tucci, C. E. M. Daily hydrological modeling in the Amazon basin using TRMM rainfall estimates. Journal of Hydrology, v.360, p. 207-216, 2008

Efron, B. Nonparametric estimates of standard error: The jackknife, the bootstrap, and other methods, Biometrika, v.68, p.589-599, 1981a.

Efron, B. Nonparametric standard erros and confidence intervals. Canadian Journal of Statistics, v.9, p.139-197, $1981 b$.

Efron, B.; Gong, G. A leisurely look at the bootstrap, the jackknife, and cross validation. American Statistician, v.37, p.36-48, 1983.

Feidas, H. Validation of satellite rainfall products over Greece. Theoretical and Applied Climatology, v.99, p.193-216, 2010.

Gonçalves, C.; Pedro Júnior, M. J.; Castro, C. E. F. Fenologia e estimativa da duração do ciclo da zínia 'Profusion Cherry' cultivada em casos em ambiente protegido. Bragantia, v.67, p.527-532, 2008.

Javanmard, S.; Yatagi, A.; Nodzu, M. I.; Bodagh, J.; Kawamoto, $\mathrm{H}$. Comparing high-resolution gridded precipitation data with satellite rainfall estimates of TRMM_3B42 over Iran. Advances in Geosciences, v.25, p.119-125, 2010.

Karaseva, M.; Prakash, S.; Gairola, R. Validation of highresolution TRMM-3B43 precipitation product using rain gauge measurements over Kyrgyzstan. Theoretical and Applied Climatology, v.108, p.147-157, 2011. 
Klering, E.V. Avaliação do uso de imagens MODIS na modelagem agrometeorológica-espectral de rendimento de arroz irrigado no Rio Grande do Sul. Porto Alegre: UFRGS, 2007. 116p. Dissertação Mestrado

Kummerow, C.; Simpson, J.; Thiele, O. The status of the Tropical Rainfall Measuring Mission (TRMM) after two years in orbit. Journal of Applied Meteorology, v.39, p.19651982, 2000.

Kurtzman, D.; Navon, S.; Morin, E. Improving interpolation of daily precipitation for hydrologic modelling: Spatial patterns of preferred interpolators. Hydrological Processes, v.23, p.3281-3291, 2009.

Latorre, M. L.; Anderson, L. O.; Shimabukuro, Y. E.; Carvalho Júnior, O. A. de. Sensor MODIS: Características gerais e aplicações. Revista Espaço e Geografia, v.6, p.91-121, 2003.

Legates, D. R.; Mccabe, G. J. Evaluating the use of 'goodnessof-fit' measures in hydrologic and hydroclimatic model validation. Water Resources Research, v.35, p.233-241, 1999.

Lin, S.; Moore, N. J.; Messina, J. P.; Visser, M. H. de; Wu, J. Evaluation of estimating daily maximum and minimum air temperature with MODIS data in east Africa. International Journal of Applied Earth Observation and Geoinformation, v.18, p.128-140, 2012.

Moraes, A. V. C.; Camargo, M. B. P.; Mascarelhas, H. A. A.; Miranda, M. A. C.; Pereira J. C. V. N. A. Teste e análise de modelos agrometeorológicos de estimativa de produtividade para a cultura da soja na região de Ribeirão Preto. Bragantia, v.67, p.527-532, 2008.
Picoli, M. C. A.; Rudorff, B. F. T.; Rizzi, R.; Giarolla, A. Índice de vegetação do sensor modis na estimativa da produtividade agrícola da cana-de-açúcar. Bragantia, v.64, p.473-483, 2009.

Running, S. W.; Justice, C. O.; Salomonson, V. Terrestrial remote sensing science and algorithms planned for EOS/ MODIS. International Journal of Remote Sensing, v.15, p.3587 -3620, 1994.

Shepherd, J. M.; Pierce, H.; Negri, A. J. Rainfall modification by major urban areas: observations from space borne rain radar on the TRMM satellite. Journal of Applied Meteorology, v.41, p.689-701, 2002.

Souza, J. D.; Silva, B. B. da. Correção atmosférica para temperatura da superfície obtida com imagem TM - Landsat 4. Revista Brasileira de Geofísica, v.23, p.349-358, 2005.

Tomlinson, C. J.; Chapman, L.; Thornes, J. E.; Baker, C. J.; Prieto-Lopez, T. Comparing night-time satellite land surface temperature from MODIS and ground measured air temperature across a conurbation. Remote Sensing Letters, v.3, p.657-666, 2012.

Wan, Z. Modis land-surface temperature algorithm theoretical basis document (LST ATBD), version 3.3. Santa Barbara: UCLA, 1999. 75p.

Wilks, D. S. Theoretical probability distributions. In: Statistical methods in the atmospheric sciences. San Diego: Academic Press, 2006. p.102-104.

Willmott, C. J. On the validation of models. Physical Geography, v.2, p.184-194, 1981.

Willmott, C. J.; Ackleson, S. G.; Davis, J. J.; Feddema, K. M.; Klink, D. R. Statistics for the evaluation and comparison of models. Journal of Geophysical Research, v.90, p.8995$9005,1985$. 